\title{
A Response to Yiasemidou et al. (2017) A Multispecialty Evaluation of Thiel Cadavers for Surgical Training
}

\author{
Anoopama Ramjeeawon ${ }^{1} \cdot$ David J. Wilson $^{1}$ (1)
}

Published online: 18 July 2017

(c) Société Internationale de Chirurgie 2017

To the Editor,

We read with interest the evaluation by Yiasemidou and her colleagues of Thiel-embalmed cadavers and their suitability for surgical training [1]. Simulation training is an increasingly important aspect of surgical training because reforms over the last 20 years have resulted in a reduction in anatomy teaching, as well as training time available to surgeons [2]. Some medical schools have moved away from cadaveric dissection [3], and this may result in junior doctors lacking an adequate knowledge of anatomy, as well as reduce the interest in surgery amongst medical students. Attaining proficiency of technical and non-technical skills needed in surgery is critical to patient safety, and inadequacy of surgical skills training in the UK may be reflected by the increasing use by surgical trainees and medical students of opportunities to travel abroad for surgical skills courses. The laws regarding dissection and surgical training using in vivo animal models are relatively relaxed in continental Europe compared to the UK, thereby allowing practice of surgical skills and procedures using models that better simulate visual and haptic qualities of living tissues. Yiasemidou et al. suggest that animal models may be considered an alternative to cadaveric simulation but lack of anatomical similarity and legal issues renders it an unlikely option in the UK [1].

David J. Wilson

wilsondj2@cf.ac.uk

1 Centre for Medical Education, College of Biomedical and Life Sciences, Cardiff University School of Medicine, Cochrane Building, Heath Park, Cardiff CF14 4XN, Wales, UK
Thiel cadaver training has many positives, but there are there some drawbacks in addition to the inadequate preservation of neurological tissues, vessel collapse and absence of bleeding reported in the evaluation. Infrastructure set-up costs are high, the cadaver loses hair and nails, the epidermis sloughs off causing the cadaver to look pale and unwrinkled; the cadaver exhibits unusual hyper-flexibility, and tissue preservation for histology is poor. Despite this, there are an increasing number of medical schools either introducing this method of preservation or considering it. This is good news for both anatomy teaching and surgical training: increased availability of high-fidelity models will improve anatomy teaching and in turn generate interest amongst students in a future career in surgery.

\section{References}

1. Yiasemidou M, Roberts D, Glassman D et al (2017) A multispecialty evaluation of Thiel cadavers for surgical training. World $\mathrm{J}$ Surg 41:1201-1207. doi:10.1007/s00268-016-3868-4

2. Standring S, Larvin M (2011) Anatomy teaching: the cruellest cut-a progress report. Bull R Coll Surg Engl 93:16-18

3. Standring S (2016) A brief history of topographical anatomy. J Anat 229:32-62 